\title{
Butterflies (Lepidoptera: Hesperioidea and Papilionoidea) of Porto Mauá, Upper Paraná Atlantic Forest Ecoregion, Rio Grande do Sul State, Brazil
}

\author{
Sabrina Campos Thiele ${ }^{1}$, Oscar Milcharek ${ }^{2}$, Fábio Luis dos Santos ${ }^{3}$ \& Lucas Augusto Kaminski ${ }^{4,5}$ \\ ${ }^{1}$ PPG-Biologia Animal, Departamento de Zoologia, Universidade Federal do Rio Grande do Sul - UFRGS, \\ Av. Bento Gonçalves, 9500, CEP 91501-970, Porto Alegre, Rio Grande do Sul, Brazil. \\ ${ }^{2}$ Rua Caxias, 1376, Centro, CEP 98900-000, Santa Rosa, Rio Grande do Sul, Brazil. \\ ${ }^{3}$ PPG-Entomologia, Departamento de Zoologia, Universidade Federal do Paraná- UFPR, C.P. 19020, \\ CEP 81531-980, Curitiba, Paraná, Brazil. \\ ${ }^{4}$ Departamento de Biologia Animal, Universidade Estadual de Campinas - UNICAMP, C.P. 6109, \\ CEP 13.083-970, Campinas, São Paulo, Brazil. \\ ${ }^{5}$ Corresponding author: Lucas Augusto Kaminski, e-mail: lucaskaminski@yahoo.com.br
}

\begin{abstract}
THIELE, S.C., MILCHAREK, O., SANTOS, F.L. \& KAMINSKI, L.A. Butterflies (Lepidoptera: Hesperioidea and Papilionoidea) of Porto Mauá, Upper Paraná Atlantic Forest Ecoregion, Rio Grande do Sul State, Brazil. Biota Neotropica. 14(2): e20130006. http://dx.doi.org/10.1590/1676-06032014000613
\end{abstract}

\begin{abstract}
This paper presents a list of species of butterflies (Lepidoptera: Hesperioidea and Papilionoidea) sampled in Porto Mauá municipality $\left(27^{\circ} 34^{\prime} \mathrm{S}, 28^{\circ} 40^{\prime} \mathrm{W}\right)$, Rio Grande do Sul State, Brazil. Sampling was carried out monthly between March 2008 and March 2009. After 204 net-hours of sampling effort, a total of 1,993 individuals from 253 species were recorded. With a single additional expedition, eight new species were added, reaching a total of 261 species recorded in the region of Porto Mauá. These new reports and the species accumulation curves may indicate a much richer fauna. The distribution of richness among butterfly families is compared with other inventories in seasonal semideciduous forest areas in the Atlantic Forest. We also discuss the importance of riparian forests of the Uruguay River as an ecological corridor that enables the maintenance of the butterfly fauna on the southern edge of the Upper Paraná Atlantic Forest Ecoregion.
\end{abstract}

Keywords: Atlantic Forest, connectivity, conservation, seasonal semi-deciduous forest, species richness.

THIELE, S.C., MILCHAREK, O., SANTOS, F.L. \& KAMINSKI, L.A. Borboletas (Lepidoptera: Hesperioidea e Papilionoidea) de Porto Mauá, Ecorregião Florestas do Alto Paraná, Rio Grande do Sul, Brasil. Biota Neotropica. 14(2): e20130006. http://dx.doi.org/10.1590/1676-06032014000613

Resumo: Este estudo apresenta uma lista de espécies de borboletas (Lepidoptera: Hesperioidea e Papilionoidea) para o município de Porto Mauá (27³4’S, $\left.28^{\circ} 40^{\prime} \mathrm{W}\right)$, Rio Grande do Sul, Brasil. As amostragens foram realizadas mensalmente, entre março de 2008 e março de 2009. Após 204 horas/rede de esforço amostral foram amostrados 1.993 indivíduos pertencentes a 253 espécies. Com apenas uma coleta adicional foram obtidos oito novos registros, totalizando 261 espécies para a região de Porto Mauá. Estes novos registros somados às curvas de acumulação de espécies indicam que a riqueza de borboletas pode ser ainda mais alta. A distribuição da riqueza entre as famílias de borboletas é comparada com inventários em áreas de floresta estacional semidecidual na Mata Atlântica. Além disso, discute-se a importância das matas ciliares do Rio Uruguai como um corredor ecológico, permitindo a manutenção da fauna de borboletas no limite sul da Ecorregião Florestas do Alto Paraná.

Palavras-chave: Conectividade, conservação, floresta estacional semidecidual, Mata Atlântica, riqueza de espécies.

\section{Introduction}

The Atlantic Forest is a hotspot of biodiversity (Mittermeier et al. 2005) consisting of a complex of 15 ecoregions sharing the same biogeographic history with similar flora and fauna. This biome extends along the Brazilian coastal region to eastern Paraguay and northeastern Argentina (Olson \& Dinerstein 1998, Di Bitetti et al. 2003). Among the 15 ecoregions, the Upper Paraná Atlantic Forest once covered the largest original area $\left(471,204 \mathrm{~km}^{2}\right)$, extending from the western slope of the Serra do Mar mountains in Brazil to eastern Paraguay and the Misiones Province in Argentina (Di Bitetti et al. 2003, Figure 1A). This area was originally covered by a continuous semi-deciduous forest with high biodiversity rates. Nowadays, this ecoregion has the largest area of remaining forest and is home to a large part of the original biota, including several endangered species (Di Bitetti et al. 2003, Ruschel et al. 2007). 

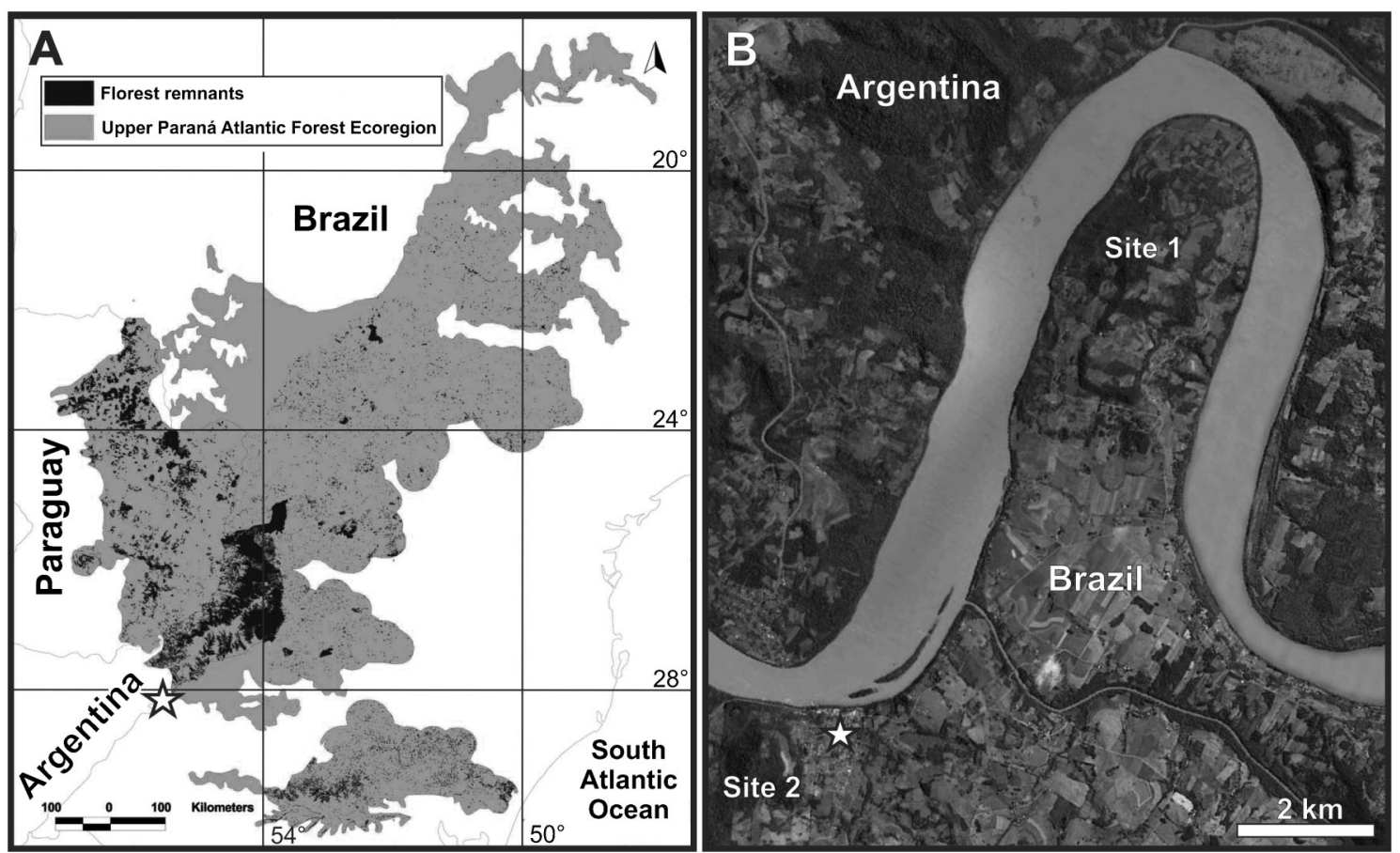

Figure 1. A, location of Porto Mauá municipality (star), Rio Grande do Sul State, Brazil, in relation to the Upper Paraná Atlantic Forest (modified from Di Bitetti et al. 2003); B, satellite image showing the urban area (star), the study sites (Site $1\left(27^{\circ} 31^{\prime} \mathrm{S}, 54^{\circ} 40^{\prime} \mathrm{W}\right)$ and Site $2\left(27^{\circ} 34^{\prime} \mathrm{S}^{\prime}\right.$; $\left.54^{\circ} 40^{\prime} \mathrm{W}\right)$ ), and the Uruguay River (modified from Google Earth).

Species inventories are the foundation for the understanding of biodiversity and the distribution pattern of species, and they provide support for actions on conservation and management, which are especially important in areas undergoing rapid environmental degradation (Lewinsohn et al. 2005). Although the butterfly fauna of the Atlantic Forest is relatively well known (see Brown \& Freitas 2000, Santos et al. 2008), in some regions the knowledge is still incipient or much localized. Moreover, the historical of anthropogenic pressure in this biome make it indispensable to perform continuous inventory studies in these areas, in which the native forest containing many endemic and/or threatened species is being replaced by monocultures (Freitas \& Marini-Filho 2011, Freitas et al. 2011, 2012). This situation is even more visible in southern Brazil (Coelho 2000, Rambo 2005), where the few existing forest remnants are limited to riparian forests and protected areas (SOS Mata Atlântica 2008).

Although the published inventories for the Upper Paraná Atlantic Forest Ecoregion (UPAF) report a richer butterfly fauna (e.g. Mielke \& Casagrande 1997, Canals 2003, NúñezBustos 2008, 2009, Núñez-Bustos et al. 2011, Francini et al. 2011), relatively few such inventories have been published, most of them for the southern boundary of this ecoregion. Our aim is to provide a list of butterflies for Porto Mauá, expanding the available knowledge about the distribution of this group of Lepidoptera at the southern border of the UPAF.

\section{Material and Methods}

\section{Study site}

Collections were carried out in Porto Mauá municipality ( $\left.27^{\circ} 34^{\prime} \mathrm{S}, 28^{\circ} 40^{\prime} \mathrm{W}\right)$, in the northwestern part of Rio Grande do Sul State (RS), Brazil (Figure 1). Porto Mauá has a total area of
$106 \mathrm{~km}^{2}$, in which the predominant forest type is seasonal semideciduous forest. Currently, $13.75 \mathrm{~km}^{2}(12.97 \%)$ of this area is covered by forest, only $4.02 \mathrm{~km}^{2}$ of it $(3.79 \%)$ in fragments larger than $0.05 \mathrm{~km}^{2}$ (IBGE 2006, SOS Mata Atlântica 2008, see also Figure 1B). The climate is subtropical humid, with the absence of a dry period (Mota 1951). Rainfall is well distributed throughout the year, with annual averages of $1,960 \mathrm{~mm}$ to $1,990 \mathrm{~mm}$ (IBGE 2006). Average temperatures are $31-34^{\circ} \mathrm{C}$ in summer and $6-9^{\circ} \mathrm{C}$ in winter (INMET 2009).

\section{Sampling}

In order to maximize species richness and describe the community of butterflies, we chose two sites adjacent to the Uruguay River (Figure 1B). Four transects were selected at these sites, including vegetation with different levels of succession and degrees of anthropogenic activity (Figure 2). Samplings were carried out monthly between March 2008 and March 2009. Each transect was sampled by two collectors with entomological nets, between 8:00 and 18:00, according to standardized sampling efforts of 1.5 hours, totaling 12 nethours per day on each month. The sequence of transects was alternated in each monthly sampling to ensure that each one would be sampled at different times of day. Individuals were captured, identified in the field and/or collected for identification.

\section{Data analysis}

The specimens were mounted and identified by consulting specialized literature, collections, and experts. The higher level classification used here follows Lamas (2004), Mielke (2005), and Wahlberg et al. (2009), with nomenclatural updates when necessary. The specimens were deposited in the following collections: Departamento de Zoologia, Universidade Federal 

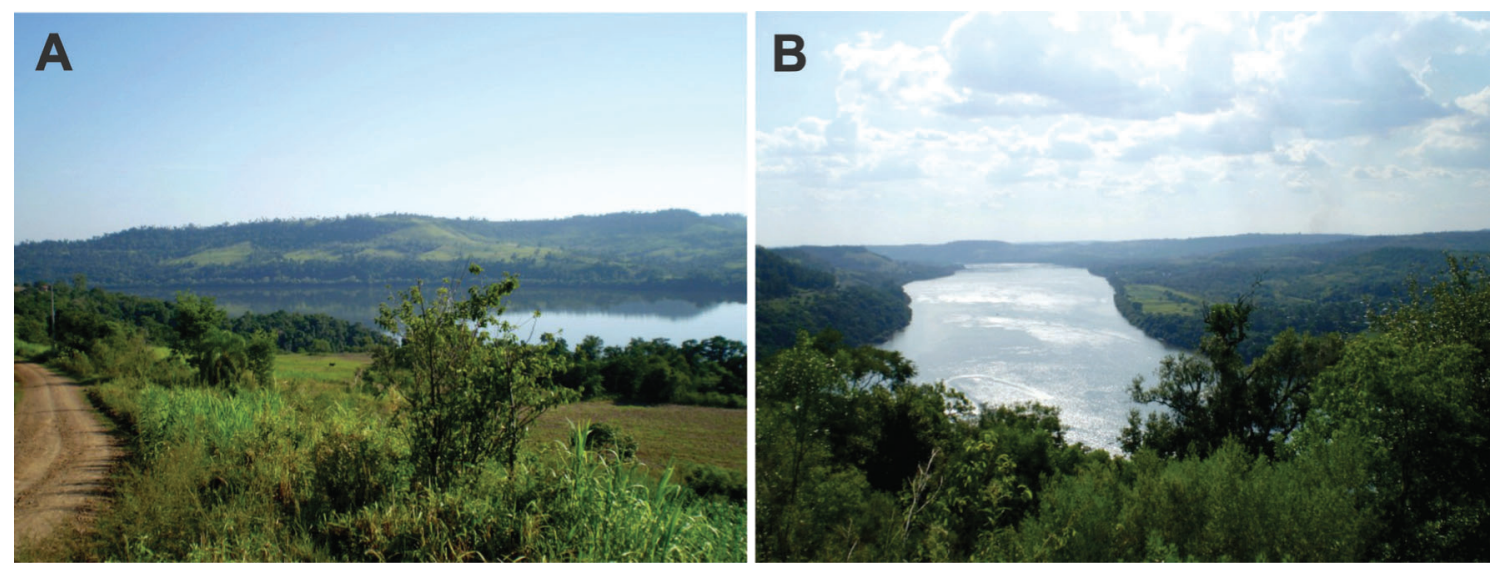

Figure 2. General view of the study sites in Porto Mauá municipality, Rio Grande do Sul State, Brazil. A, detail of Site 1, showing the road, pastures and riparian vegetation on the Brazilian side, and the Argentinean side on the bottom; B, view from the top of a hill in Site 2, showing the Uruguay River.

do Rio Grande do Sul (UFRGS), Porto Alegre, Rio Grande do Sul State, Brazil; Coleção Entomológica Pe. Jesus Santiago Moure (DZUP), Departamento de Zoologia, Universidade Federal do Paraná, Curitiba, Paraná State, Brazil; Museu de Zoologia “Adão José Cardoso" (ZUEC), Universidade Estadual de Campinas, Campinas, São Paulo State, Brazil.

Abundance (N) and richness (S) of butterflies were analyzed. Species acumulation curve was also plotted, including the observed and estimated total richness with confidence interval (95\%), using the EstimateS 8.0 software (Colwell 2007). This same procedure was also performed separately for each family. Also, we have an estimated of richness using the "Jackknife 2" estimator, which considers the abundance in the samplings. An additional sampling was conducted in May 2009, which resulted in new additions to the list (Table 1); however, because the sampling protocol is not standardized, these sampling was not included in the analysis.

\section{Results and Discussion}

After a total sampling effort of 204 net-hours, 1,993 individuals from 253 species were sampled (Table 1). In the additional sampling, eight new species were added, reaching

Table 1. Butterflies (Papilionoidea and Hesperioidea) recorded in Porto Mauá municipality, Rio Grande do Sul State, Brazil. The number of species from each major taxon is reported within parentheses. *Species collected during one additional visit. Total: 261 species.

\begin{tabular}{|c|c|c|c|c|}
\hline Families & Subfamilies & Tribes & Species & $\mathbf{N}$ \\
\hline \multirow[t]{24}{*}{ Hesperiidae (86) } & \multirow[t]{18}{*}{ Eudaminae (18) } & \multirow[t]{18}{*}{ Eudamini (18) } & Astraptes alardus alardus (Stoll, 1790) & 2 \\
\hline & & & Astraptes aulus (Plötz, 1881) & 1 \\
\hline & & & Astraptes fulgerator fulgerator (Walch, 1775) & 1 \\
\hline & & & Autochton zarex (Hübner, 1818) & 5 \\
\hline & & & Polygonus leo pallida Röber, 1925 & 1 \\
\hline & & & Polygonus savigny savigny (Latreille, [1824]) & 3 \\
\hline & & & Spathilepia clonius (Cramer, 1775) & 1 \\
\hline & & & Urbanus albimargo rica Evans, 1952 & 2 \\
\hline & & & Urbanus dorantes dorantes (Stoll, 1790) & 18 \\
\hline & & & Urbanus doryssus albicuspis (Herrich-Schäffer, 1869) & 1 \\
\hline & & & Urbanus esta Evans, 1952 & 1 \\
\hline & & & Urbanus evenus (Ménétriés, 1855) & 3 \\
\hline & & & Urbanus pronta Evans, 1952 & 2 \\
\hline & & & Urbanus proteus proteus (Linnaeus, 1758) & 1 \\
\hline & & & Urbanus simplicius (Stoll, 1790) & 5 \\
\hline & & & Urbanus sp. & 1 \\
\hline & & & Urbanus teleus (Hübner, 1821) & 6 \\
\hline & & & Urbanus virescens (Mabille, 1877) & 3 \\
\hline & \multirow[t]{6}{*}{ Hesperiinae (40) } & \multirow[t]{3}{*}{ Anthoptini (03) } & Anthoptus epictetus (Fabricius, 1793) & 2 \\
\hline & & & Corticea corticea (Plötz, 1882) & 3 \\
\hline & & & Synapte silius (Latreille, [1824]) & 9 \\
\hline & & \multirow[t]{3}{*}{ Calpodini (04) } & Evansiella cordela (Plötz, 1882) & 2 \\
\hline & & & Lychnuchoides ozias ozias (Hewitson, 1878) & 6 \\
\hline & & & Saliana longirostris (Sepp, [1840]) & 1 \\
\hline
\end{tabular}


Table 1. Continued.

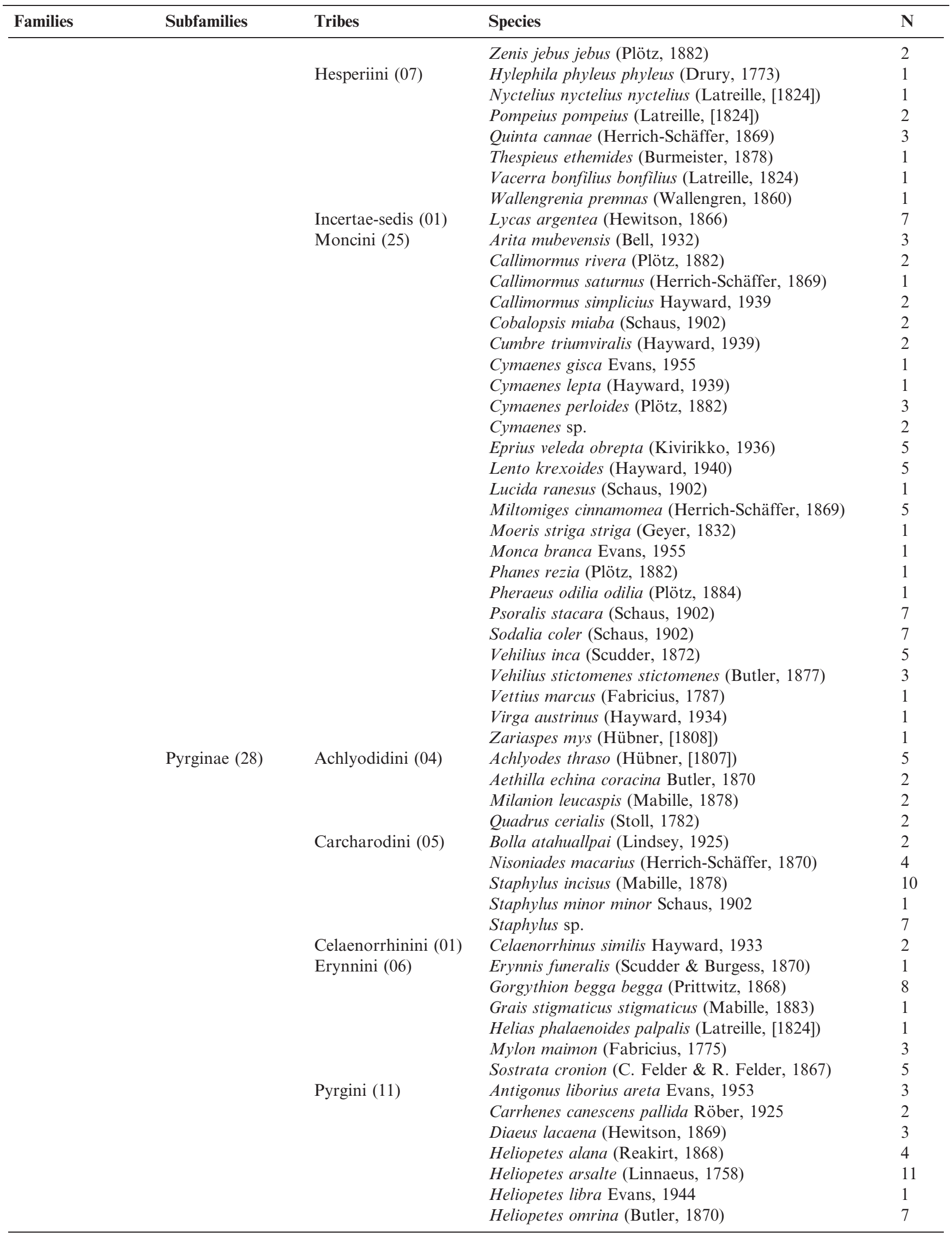


Table 1. Continued.

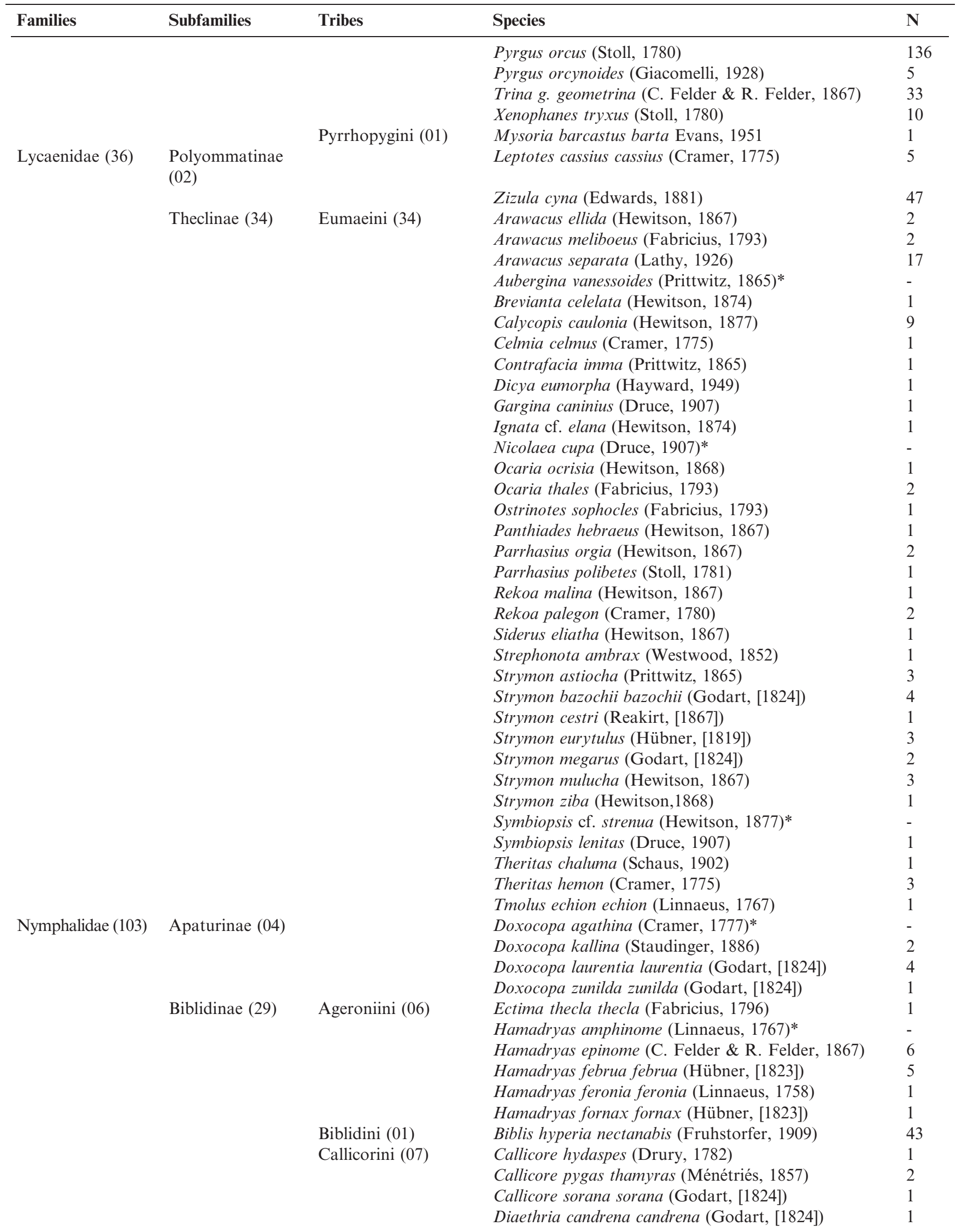


Thiele, S.C. et al.

Table 1. Continued.

\begin{tabular}{|c|c|c|c|c|}
\hline Families & Subfamilies & Tribes & Species & $\mathbf{N}$ \\
\hline & & \multirow{6}{*}{ Catonephelini (03) } & Diaethria clymena janeira (C. Felder, 1862) & 1 \\
\hline & & & Haematera pyrame pyrame (Hübner, [1819]) & 2 \\
\hline & & & Paulogramma pyracmon pyracmon (Godart, [1824]) & 1 \\
\hline & & & Cybdelis phaesyla (Hübner, [1831]) & 1 \\
\hline & & & Eunica eburnea Fruhstorfer, 1907 & 1 \\
\hline & & & Myscelia orsis (Drury, 1782) & 3 \\
\hline & & \multirow[t]{4}{*}{ Epiphelini (04) } & Epiphile hubneri Hewitson, 1861 & 3 \\
\hline & & & Epiphile orea orea (Hübner, [1823]) & 1 \\
\hline & & & Pyrrhogyra neaerea arge Gosse, 1880 & 2 \\
\hline & & & Temenis laothoe meridionalis Ebert, 1965 & 1 \\
\hline & & \multirow[t]{8}{*}{ Eubagini (08) } & Dynamine aerata (Butler, 1877) & 2 \\
\hline & & & Dynamine agacles agacles (Dalman, 1823) & 10 \\
\hline & & & Dynamine artemisia artemisia (Fabricius, 1793) & 1 \\
\hline & & & Dynamine athemon athemaena (Hübner, [1824]) & 9 \\
\hline & & & Dynamine coenus coenus (Fabricius, 1793) & 2 \\
\hline & & & Dynamine myrrhina (Doubleday, 1849) & 29 \\
\hline & & & Dynamine postverta postverta (Cramer, 1779$)$ & 15 \\
\hline & & & Dynamine tithia tithia (Hübner, [1823]) & 21 \\
\hline & \multirow[t]{3}{*}{ Charaxinae (03) } & \multirow[t]{3}{*}{ Anaeini (03) } & Fountainea ryphea phidile (Geyer, 1837) & 2 \\
\hline & & & Hypna clytemnestra huebneri Butler, 1866 & 2 \\
\hline & & & Memphis acidalia victoria (H. Druce, 1877) & 4 \\
\hline & \multirow[t]{2}{*}{ Cyrestinae (02) } & & Marpesia chiron marius (Cramer, 1779) & 3 \\
\hline & & & Marpesia petreus petreus (Cramer, 1776) & 1 \\
\hline & \multirow[t]{19}{*}{ Danainae (19) } & \multirow[t]{2}{*}{ Danaini (02) } & Danaus erippus (Cramer, 1775) & 44 \\
\hline & & & Danaus gilippus gilippus (Cramer, 1775) & 5 \\
\hline & & \multirow[t]{8}{*}{ Ithomiini (08) } & Dircenna dero celtina Burmeister, 1878 & 12 \\
\hline & & & Episcada carcinia Schaus, 1902 & 1 \\
\hline & & & Episcada hymenaea hymenaea (Prittwitz, 1865) & 20 \\
\hline & & & Epityches eupompe (Geyer, 1832) & 2 \\
\hline & & & Mechanitis lysimnia lysimnia (Fabricius, 1793) & 4 \\
\hline & & & Placidina euryanassa (C. Felder \& R. Felder, 1860) & 6 \\
\hline & & & Pseudoscada erruca (Hewitson, 1855) & 10 \\
\hline & & & Pteronymia sylvo (Geyer, 1832) & 6 \\
\hline & & Acraeini (01) & Actinote melanisans Oberthür, 1917 & 1 \\
\hline & & Argynnini (01) & Euptoieta hegesia meridiania Stichel, 1938 & 1 \\
\hline & & \multirow[t]{7}{*}{ Heliconiini (07) } & Agraulis vanillae maculosa (Stichel, [1908]) & 5 \\
\hline & & & Dione juno juno (Cramer, 1779) & 2 \\
\hline & & & Dryas iulia alcionea (Cramer, 1779) & 32 \\
\hline & & & Eueides aliphera aliphera (Godart, 1819) & 26 \\
\hline & & & Eueides isabella dianasa (Hübner, [1806]) & 1 \\
\hline & & & Heliconius erato phyllis (Fabricius, 1775) & 68 \\
\hline & & & Heliconius ethilla narcaea (Godart, 1819) & 3 \\
\hline & Libytheinae $(01)$ & & Libytheana carinenta carinenta (Cramer, 1777) & 1 \\
\hline & \multirow[t]{5}{*}{ Limenitidinae (05) } & & Adelpha abia (Hewitson, 1850) & 1 \\
\hline & & & Adelpha epizygis epizygis Fruhstorfer, 1915* & - \\
\hline & & & Adelpha malea goyama Schaus, 1902* & - \\
\hline & & & Adelpha syma (Godart, [1824]) & 1 \\
\hline & & & Adelpha thessalia indefecta Fruhstorfer, 1913 & 1 \\
\hline & \multirow[t]{9}{*}{ Nymphalinae (13) } & Junoniini (01) & Junonia evarete flirtea (Fabricius, 1793) & 118 \\
\hline & & \multirow[t]{7}{*}{ Melitaeini (07) } & Chlosyne lacinia saundersi (Doubleday, [1847]) & 5 \\
\hline & & & Eresia lansdorfi (Godart, 1819) & 5 \\
\hline & & & Ortilia dicoma (Hewitson, 1864) & 3 \\
\hline & & & Ortilia ithra (Kirby, 1900) & 26 \\
\hline & & & Ortilia orthia (Hewitson, 1864) & 7 \\
\hline & & & Ortilia velica durnfordi (Godman \& Salvin, 1878) & 6 \\
\hline & & & Tegosa claudina (Eschscholtz, 1821) & 140 \\
\hline & & Nymphalini (03) & Hypanartia bella (Fabricius, 1793) & 13 \\
\hline
\end{tabular}


Table 1. Continued.

\begin{tabular}{|c|c|c|c|c|}
\hline Families & Subfamilies & Tribes & Species & $\mathbf{N}$ \\
\hline & \multirow{31}{*}{ Satyrinae (27) } & \multirow{4}{*}{ Victorinini (02) } & Hypanartia lethe lethe (Fabricius, 1793) & 9 \\
\hline & & & Vanessa braziliensis (Moore, 1883) & 40 \\
\hline & & & Anartia amathea roeselia (Eschscholtz, 1821) & 46 \\
\hline & & & Siproeta epaphus trayja Hübner, [1823] & 14 \\
\hline & & \multirow[t]{2}{*}{ Brassolini (02) } & Caligo illioneus pampeiro Fruhstorfer, 1904 & 1 \\
\hline & & & Narope cyllastros E. Doubleday, [1849] & 1 \\
\hline & & Melanitini (01) & Manataria hercyna hercyna (Hübner, [1821]) & 1 \\
\hline & & Morphini (01) & Morpho helenor achillides C. Felder \& R. Felder, 1867 & 49 \\
\hline & & \multirow[t]{23}{*}{ Satyrini (23) } & Carminda griseldis (Weymer, 1911)* & - \\
\hline & & & Eteona tisiphone (Boisduval, 1836) & 5 \\
\hline & & & Forsterinaria necys (Godart, [1824]) & 11 \\
\hline & & & Forsterinaria quantius (Godart, [1824]) & 12 \\
\hline & & & Godartiana muscosa (Butler, 1870) & 4 \\
\hline & & & Hermeuptychia atalanta (Butler, 1867) & 67 \\
\hline & & & Moneuptychia griseldis (Weymer, 1911) & 2 \\
\hline & & & Pareuptychia ocirrhoe interjecta (d'Almeida, 1952) & 1 \\
\hline & & & Pareuptychia summandosa (Gosse, 1880) & 29 \\
\hline & & & Paryphthimoides eous (Butler, 1867) & 16 \\
\hline & & & Paryphthimoides grimon (Godart, [1824]) & 3 \\
\hline & & & Paryphthimoides phronius (Godart, [1824]) & 76 \\
\hline & & & Paryphthimoides poltys (Prittwitz, 1865) & 2 \\
\hline & & & Pharneuptychia pharnabazos (Bryk, 1953) & 1 \\
\hline & & & Praepedaliodes phanias (Hewitson, 1862) & 24 \\
\hline & & & Pseudodebis euptychidia (Butler, 1868) & 1 \\
\hline & & & Splendeuptychia libitina (Butler, 1870) & 11 \\
\hline & & & Taygetis tripunctata Weymer, 1907 & 2 \\
\hline & & & Taygetis ypthima Hübner, [1821] & 10 \\
\hline & & & Yphthimoides celmis (Godart, [1824]) & 9 \\
\hline & & & Yphthimoides mimula (Hayward, 1954) & 2 \\
\hline & & & $\begin{array}{l}\text { Yphthimoides ordinaria Freitas, Kaminski \& Mielke, } \\
2012\end{array}$ & 10 \\
\hline & & & Yphthimoides straminea (Butler, 1867) & 1 \\
\hline \multirow[t]{8}{*}{ Papilionidae (08) } & \multirow[t]{8}{*}{ Papilioninae (08) } & & Battus polydamas polydamas (Linnaeus, 1758) & 11 \\
\hline & & & Battus polystictus polystictus (Butler, 1874) & 4 \\
\hline & & & Heraclides anchisiades capys (Hübner, [1809]) & 1 \\
\hline & & & Heraclides astyalus astyalus (Godart, 1819) & 6 \\
\hline & & & Heraclides hectorides (Esper, 1794) & 6 \\
\hline & & & $\begin{array}{l}\text { Heraclides thoas brasiliensis (Rothschild \& Jordan, } \\
\text { 1906) }\end{array}$ & 2 \\
\hline & & & Parides agavus (Drury, 1782) & 28 \\
\hline & & & Parides anchises nephalion (Godart, 1819) & 9 \\
\hline \multirow[t]{14}{*}{ Pieridae (14) } & \multirow[t]{10}{*}{ Coliadinae (10) } & & Aphrissa statira statira (Cramer, 1777) & 8 \\
\hline & & & Eurema albula sinoe (Godart, 1819) & 2 \\
\hline & & & Eurema deva deva (Doubleday, 1847) & 29 \\
\hline & & & Eurema elathea flavescens (Chavannes, 1850) & 2 \\
\hline & & & Phoebis argante argante (Fabricius, 1775) & 11 \\
\hline & & & Phoebis neocypris neocypris (Hübner, [1823]) & 19 \\
\hline & & & Phoebis philea philea (Linnaeus, 1763) & 7 \\
\hline & & & Phoebis sennae marcellina (Cramer, 1777) & 12 \\
\hline & & & Pyrisitia leuce leuce (Boisduval, 1836) & 2 \\
\hline & & & Rhabdodryas trite banksi (Breyer, 1939) & 4 \\
\hline & \multirow[t]{3}{*}{ Dismorphinae (03) } & & Dismorphia astyocha (Hübner, [1831]) & 1 \\
\hline & & & Enantia lina psamathe (Fabricius, 1793) & 3 \\
\hline & & & Pseudopieris nehemia nehemia (Boisduval, 1836) & 6 \\
\hline & Pierinae $(01)$ & & Ascia monuste orseis (Godart, 1819) & 3 \\
\hline \multirow[t]{2}{*}{ Riodinidae (14) } & Euselasiinae $(01)$ & Euselasiini (01) & Euselasia eucerus (Hewitson, 1872) & 1 \\
\hline & Riodininae (13) & Incertae-sedis (03) & Emesis diogenia Prittwitz, 1865 & 9 \\
\hline
\end{tabular}


Table 1. Continued.

\begin{tabular}{|c|c|c|c|c|}
\hline Families & Subfamilies & Tribes & Species & $\mathbf{N}$ \\
\hline & & & Emesis ocypore zelotes Hewitson, 1872 & 5 \\
\hline & & & Emesis satema (Schaus, 1902) & 2 \\
\hline & & Mesosemiini (01) & Ionotus alector (Geyer, 1837) & 2 \\
\hline & & \multirow{2}{*}{ Nymphidiini (02) } & Adelotypa argiella Bates, 1868 & 1 \\
\hline & & & Synargis calyce (C. Felder \& R. Felder, 1862) & 1 \\
\hline & & \multirow[t]{7}{*}{ Riodinini (07) } & Barbicornis basilis mona Westwood, 1851 & 4 \\
\hline & & & Calephelis braziliensis McAlpine, 1971 & 2 \\
\hline & & & Calephelis aymaran McAlpine, 1971 & 2 \\
\hline & & & Caria marsyas Godman, 1903 & 1 \\
\hline & & & Chalodeta theodora (C. Felder \& R. Felder, 1862) & 3 \\
\hline & & & Lasaia agesilas agesilas (Latreille, [1809]) & 1 \\
\hline & & & Melanis xenia xenia (Hewitson, [1853]) & 2 \\
\hline
\end{tabular}
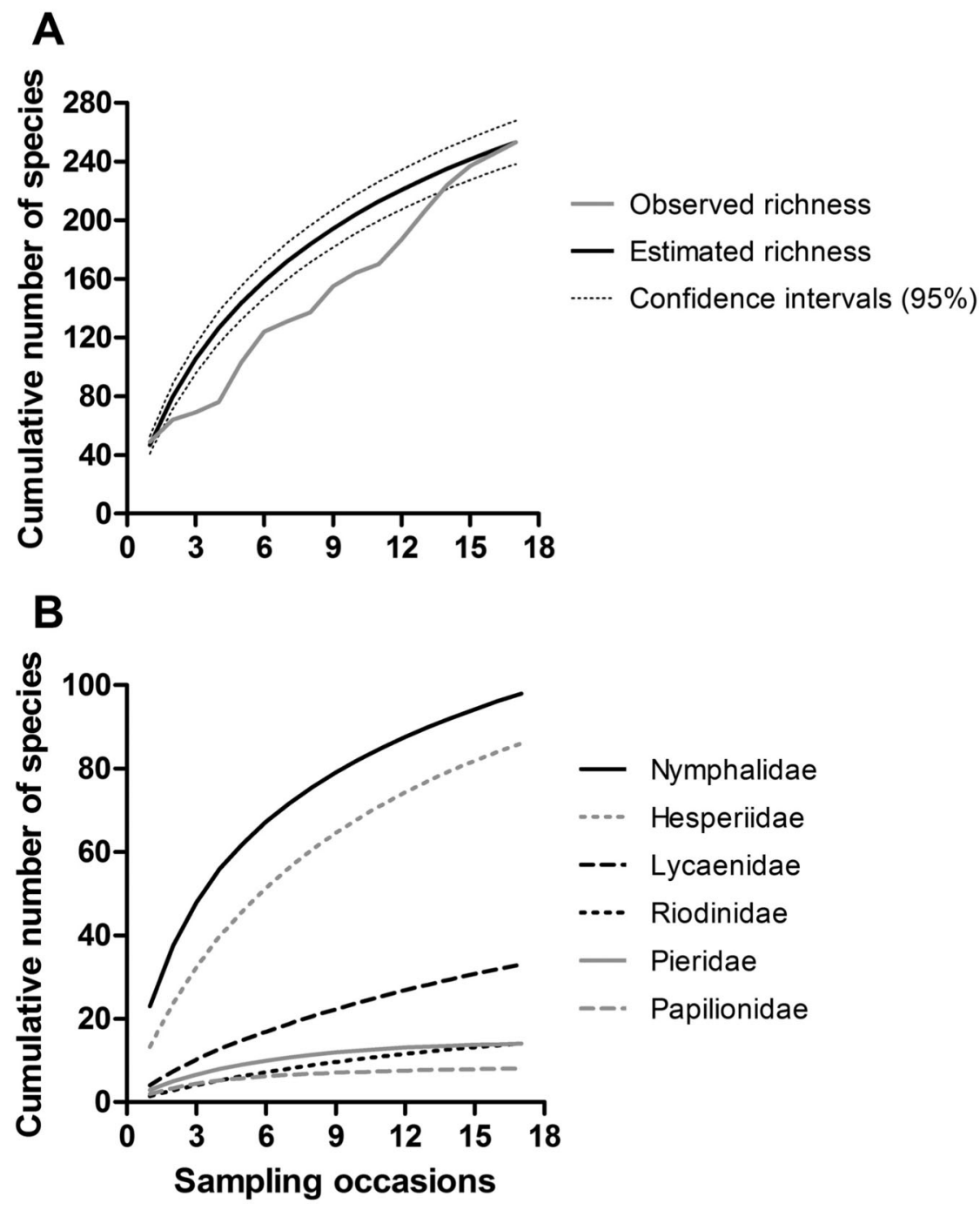

Figure 3. Cumulative number of species recorded after 17 sampling occasions in Porto Mauá municipality, Rio Grande do Sul State, Brazil, from March 2008 to March 2009. A, total cumulative richness for observed and expected curves with confidence intervals of $95 \%$; B, cumulative number of species per butterfly family. 
Butterflies of Porto Mauá

Table 2. Comparison of butterfly richness and composition in locations with predominance of semi-deciduous forest in the Upper Paraná Atlantic Forest Ecoregion. The relative percentages are reported in parentheses.

\begin{tabular}{|c|c|c|c|c|c|c|c|c|}
\hline Localities & NYM & HESP & LYC & RIO & PIE & PAP & Total & Sampling effort \\
\hline Porto Mauá (RS) 1 & $103(39.5)$ & $86(33)$ & $36(13.8)$ & $14(5.3)$ & $14(5.3)$ & $8(3.1)$ & 261 & 204 hours \\
\hline Northwest Region (RS) ${ }^{2}$ & $110(31.2)$ & $119(33.7)$ & $54(15.3)$ & $35(9.9)$ & $21(5.9)$ & $14(4)$ & 353 & $\sim 1000$ hours \\
\hline Frederico Westphalen $(\mathrm{RS})^{3,4}$ & $108(49.5)$ & $54(24.8)$ & $16(7.3)$ & $11(5)$ & $18(8.3)$ & $11(5)$ & 218 & 220 hours \\
\hline Val da Serra $(\mathrm{RS})^{5}$ & $54(50.5)$ & $21(19.6)$ & $9(8.4)$ & $7(6.5)$ & $9(8.4)$ & $7(6.5)$ & 107 & 105 hours \\
\hline Santa Maria (RS) ${ }^{6}$ & $51(35.2)$ & $58(40)$ & $9(6.2)$ & $7(4.8)$ & $8(5.5)$ & $12(8.3)$ & 145 & 135 hours \\
\hline $\begin{array}{l}\text { Reserva Privada Yacutinga } \\
\text { (Misiones) }\end{array}$ & $174(30.4)$ & $248(43.4)$ & $68(11.9)$ & $49(8.6)$ & $21(3.7)$ & $12(2.1)$ & 572 & 1860 hours \\
\hline $\begin{array}{l}\text { Reserva Nacional Iguazú } \\
\text { (Misiones) }^{8}\end{array}$ & $189(28.9)$ & $284(43.5)$ & $72(11)$ & $60(9.2)$ & $30(4.6)$ & $18(2.8)$ & 653 & 948 hours \\
\hline
\end{tabular}

References: 1, this study; 2, Fábio L. dos Santos (unpublished data, including records from Augusto Pestana, Catuípe, ljuí, Boa Vista do Cadeado, Bozano, and Pejuçara); 3, Giovenardi et al. (2008); 4, Bonfantti et al. (2009); 5, Morais et al. (2012); 6, Dessuy \& Morais (2007); 7 , Núñez-Bustos (2008); 8, Núñez-Bustos (2009). Butterfly families: HESP, Hesperiidae; PAP, Papilionidae; PIE, Pieridae; LYC, Lycaenidae; RIO, Riodinidae; NYM, Nymphalidae.

261 species recorded in the region of Porto Mauá. The species accumulation curve is far from stabilization with a marked ascending pattern (Figure 3), clearly indicating that the community of butterflies was not fully sampled. When compared with other inventories with more extensive sampling efforts completed in the UPAF, such as those conducted in the Province of Misiones, Argentina, with 572 species collected in Reserva Privada Yacutinga and 653 in Reserva Nacional Iguazú (Nunez-Bustos 2008, 2009), it becomes evident that the richness of butterflies of Porto Mauá should be greater. Based on the sampling sufficiency curves, and considering the richness observed in nearby well-sampled areas, i.e., with more than 1,000 net-hours (see Table 2), we expect that the complete list of butterflies of Porto Mauá includes 300 to 400 species. This prediction is confirmed by the "Jackknife 2" estimator that indicates 377 species to Porto Mauá.

Taking into account that our sampling effort represents only $11 \%$ of the effort made in Reserva Privada Yacutinga, we note that the list of butterflies obtained for Porto Mauá is rich and surpasses other nearby areas in Rio Grande do Sul (Table 2), such as Frederico Westphalen, with 220 net-hours (Giovenardi et al. 2008, Bonfantti et al. 2009), Val da Serra, with 105 net-hours (Morais et al. 2012), and Santa Maria, with 135 net-hours (Dessuy \& Morais 2007). Regarding taxonomic composition, the richest family was Nymphalidae, followed by Hesperiidae, Lycaenidae, Pieridae and Riodinidae, and Papilionidae (Table 2). Nymphalidae and Hesperiidae are commonly reported as the most representative families in Neotropical inventories (see Table 2). As proposed by Francini et al. (2011), we expect that with an increased sampling effort, the richness of Hesperiidae will outweigh Nymphalidae. We also expect the richness of Lycaenidae and Riodinidae to increase, since the populations of these butterflies vary greatly over time, requiring longer temporal surveys for adequate sampling of these families (Iserhard et al. 2013).

Some characteristics of the study area, such as its proximity to large interconnected forest fragments in Argentina, make it an important riparian corridor along the Uruguay River, since this river does not represent a barrier for most butterflies. About $80 \%$ of the species present in Porto Mauá are also found in these preserved areas of Argentina, which shows the importance of these remnants of riparian forest on the Brazilian side of the river. These data highlight the need for conservation of this area, as it is one of the last sites occupied by forest patches in the region (Figure 1A). According to Freitas (2010), the reduction of riparian forests with consequent loss of connectivity can cause serious impacts to butterfly communities, bringing on structural changes, especially in regions that are already severely modified, like the Upper Paraná Atlantic Forest. Accordingly, forest management and restoration initiatives should consider this area as key to the establishment of ecological corridors.

Our study emphasizes the importance of faunal inventories, especially in sites in which there is urgency for preservation. In the case of butterflies, species lists may provide relevant information about diversity, distribution and ecology (Motta 2002, Freitas et al. 2003), and such information can serve as the basis for ecological zoning and conservation action plans.

\section{Acknowledgements}

We thank Peter Pisoni for his kind reception and promptness during the collection expeditions, and Silvana Gasparetto for suggesting sampling areas and mediating our interactions with local residents. To Alfred Moser, André V. L. Freitas, Diego R. Dolibaina, Eduardo S. Carneiro, Fernando M. Dias, Luis A. R. Leite, Gilson R. P. Moreira, Olaf H. H. Mielke, and Noemy Seraphim for help with species identification. Special thanks to Cristiano Iserhard for discussions about butterfly ecology and statistical assistance. LAK thanks Fundação de Amparo à Pesquisa do Estado de São Paulo (FAPESP 10/51340-8), RedeLep "Rede Nacional de Pesquisa e Conservação de Lepidópteros" SISBIOTA-Brasil/CNPq (563332/2010-7), and the BIOTA FAPESP program (11/ 50225-3).

\section{References}

BONFANTTI, D., DI MARE, R.A. \& GIOVENARDI, R. 2009. Butterflies (Lepidoptera: Papilionoidea and Hesperioidea) from two forest fragments in northern Rio Grande do Sul, Brazil. Check List 5:819-829.

BROWN Jr., K.S \& FREITAS, A.V.L. 2000. Atlantic Forest butterflies: indicators for landscape conservation. Biotropica 32:934-956.

CANALS, G.R. 2003. Mariposas de Misiones. L.O.L.A., Buenos Aires, 492p.

COELHO, G.C. 2000. A floresta nativa do Noroeste do RS - questões relevantes para a conservação. Caderno de Pesquisa Sér. Bot. (Santa Cruz do Sul) 12:17-44. 
COLWELL, R.K. 2007. Estimates 8.0: statistical estimation of species richness and shared species from samples. University of Connecticut, Connecticut. http://viceroy.eeb.ucon.edu/estimates.

DESSUY, M.B. \& MORAIS, A.B.B. 2007. Diversidade de borboletas (Lepidoptera, Papilionoidea e Hesperioidea) em fragmentos de Floresta Estacional Decidual em Santa Maria, Rio Grande do Sul, Brasil. Rev. Bras. Zool. 24:108-120.

DI BITETTI, M.S., PLACCI, G \& DIETZ, L.A. 2003. A Biodiversity vision for the Upper Paraná Atlantic Forest Ecoregion: Designing a biodiversity conservation landscape and setting priorities for conservation Action. World Wildlife Fund, Washington.

FRANCINI, R.B., DUARTE, M., MIELKE, O.H.H., CALDAS, A. \& FREITAS, A.V.L. 2011. Butterflies (Lepidoptera, Papilionoidea and Hesperioidea) of the "Baixada Santista" region, coastal São Paulo, southeastern Brazil. Rev. Bras. Entomol. 55:55-68.

FREITAS, A.V.L. 2010. Impactos potenciais das mudanças propostas no Código Florestal Brasileiro sobre as borboletas. Biota Neotrop. 10:53-57.

FREITAS, A.V.L. \& MARINI-FILHO, O.J. 2011. Plano de Ação Nacional para Conservação dos Lepidópteros Ameaçados de Extinção. ICMBio, Brasília, 124p.

FREITAS, A.V.L., FRANCINI, R.B. \& BROWN Jr., K.S. 2003. Insetos como indicadores ambientais. In Métodos de estudos em biologia da conservação e manejo da vida silvestre (L. Cullen Junior, C. Valladares-Pádua \& R. Rudran, orgs.). Editora da UFPR, Curitiba, p.125-151.

FREITAS, A.V.L., KAMINSKI, L.A., ISERHARD, C.A., BARBOSA, E.P. \& MARINI-FILHO, O.J. 2011. The endangered butterfly Charonias theano (Boisduval) (Lepidoptera: Pieridae): current status, threats and its rediscovery in São Paulo state, southeastern Brazil. Neotrop. Entomol. 40:669-676.

FREITAS, A.V.L., WAHLBERG, N., MATOS-MARAVI, P.F., MARIN, M.A. \& MIELKE, O.H.H. 2012. Euptychia boulleti (Le Cerf) n. comb. (Lepidoptera: Nymphalidae: Satyrinae), a rare and endangered butterfly from Southeastern Brazil. Neotrop. Entomol. 41:461-467.

GIOVENARDI, R., DI MARE, R.A., SPONCHIADO, J., ROANI, S.H., JACOMASSA, F.A.F., JUNG, A.B. \& PORN, M.A. 2008. Diversidade de Lepidoptera (Papilionoidea e Hesperioidea) em dois fragmentos de floresta no município de Frederico Westphalen, Rio Grande do Sul, Brasil. Rev. Bras. Entomol. 52:599-605.

INMET. Normais Climatológicas 1931-1990. www.inmet.gov.br (último acesso em 23/06/2009).

INSTITUTO BRASILEIRO DE GEOGRAFIA E ESTATÍSTICA (IBGE). Cidades. Censo Agropecuário 2006. http://www.ibge.gov. br/cidadesat/default.php (último acesso em 16/06/2008).

ISERHARD, C.A., QUADROS, M.T., ROMANOWSKI, H.P. \& MENDONÇA-JUNIOR, M.S. 2010. Borboletas (Lepidoptera: Papilionoidea e Hesperioidea) ocorrentes em diferentes ambientes na Floresta Ombrófila Mista e Campos de Cima da Serra do Rio Grande do Sul, Brasil. Biota Neotrop. 10:309-320.

ISERHARD, C.A., BROWN Jr., K.S \& FREITAS, A.V.L. 2013. Maximized sampling of butterflies to detect temporal changes in tropical communities. J. Insect Conserv. 17:615-622.

LAMAS, G. 2004. Checklist: Part 4A. Hesperioidea-Papilionoidea. In Atlas of Neotropical Lepidoptera. (J.B. Heppner, ed.). Association for Tropical Lepidoptera/Scientific Publishers, Gainesville, p.1-439.

LEWINSOHN, T.M., FREITAS, A.V.L \& PRADO, P.I. 2005. Conservation of terrestrial invertebrates and their habitats in
Brazil. Conserv. Biol. 19:640-645

MARCHIORI, M.O. \& ROMANOWSKI, H.P. 2006. Borboletas (Lepidoptera: Papilionoidea e Hesperioidea) do Parque Estadual do Espinilho e seu entorno, Rio Grande do Sul, Brasil. Rev. Bras. Zool. 23:1029-1037.

MIELKE, O.H.H. 2005. Catalogue of the American Hesperioidea: Hesperiidae (Lepidoptera). Sociedade Brasileira de Zoologia, Curitiba, 1536p.

MIELKE, O.H.H. \& CASAGRANDE, M.M. 1997. Papilionoidea e Hesperioidea (Lepidoptera) do Parque Estadual do Morro do Diabo, Teodoro Sampaio, São Paulo, Brasil. Rev. Bras. Zool. 14(4):967-1001.

MITTERMEIER, R.A., GIL, R.P., HOFFMAN, M., PILGRIM, J., BROOKS, T., MITTERMEIER, C.G., LAMOREUX, J. \& FONSECA, G.A.B. 2005. Hotspots revisited: earth's biologically richest and most endangered terrestrial ecoregions, 2. ed. University of Chicago Press, Boston.

MORAIS, A.B.B., LEMES, R. \& RITTER, C.D. 2012. Borboletas (Lepidoptera: Hesperioidea e Papilionoidea) de Val da Serra, região central do Rio Grande do Sul, Brasil. Biota Neotrop 12(2):1-9 http://www.biotaneotropica.org.br/v12n2/en/abstract?inventory+bn01412022012 (último acesso em 13/12/2012).

MOTA, F.S. 1951. Estudos do clima do Estado do Rio Grande do Sul, segundo o sistema de W. Köppen. Rev. Bras. Geog. 13:275284.

MOTTA, P.C. 2002. Butterflies from the Uberlândia region, central Brazil: species list end biological comments. Braz. J. Biol. 62(1):151163.

NÚNEZ-BUSTOS, E.O. 2008. Diversidad de mariposas diurnas em la Reserva Privada Yacutinga, Provincia de Misiones, Argentina (Lepidoptera: Hesperioidea y Papilionoidea). Trop. Lepid. Res. 18:78-87.

NUÑEZ-BUSTOS, E.O. 2009. Mariposas diurnas (Lepidoptera: Papilionoidea y Hesperioidea) del Parque Nacional Iguazú, Provincia de Misiones, Argentina. Trop. Lepid. Res. 19:71-81.

NÚÑEZ-BUSTOS, E.O., FAVRE, P., BERTOLINI, M.P., TURNER, J.D. \& SOURAKOV, A. 2011. Mariposas diurnas (Lepidoptera: Papilionoidea y Hesperioidea) de la Reserva Privada OsununúParque Provincial Teyú Cuaré y alrededores de San Ignacio, Provincia de Misiones, Argentina. Trop. Lepid. Res. 21:34-42.

OLSON, D.M. \& DINERSTEIN, E. 1998. The Global 200: A representation approach to conserving the earth's most biologically valuable ecoregions. Conserv. Biol. 12:502-515.

RAMBO, B. 2005. A fisionomia do Rio Grande do Sul: ensaio de monografia natural. Unisinos, São Leopoldo.

RUSCHEL, A.R., NODARI, R.O. \& MOERSCHBACHER, B.M. 2007. Woody plant species richness in the Turvo State park, a large remnant of deciduous Atlantic forest, Brazil. Biodivers. Conserv. 16:1699-1714.

SANTOS, E.C., MIELKE, O.H.H. \& CASAGRANDE, M.M. 2008. Inventários de borboletas no Brasil: estado da arte e modelo de áreas prioritárias para pesquisa com vistas à conservação. Nat. Conservação 6:68-90.

SOS MATA ATLÂNTICA. Atlas Mata Atlântica. http://www. sosmataatlantica.org.br/ (último acesso em 06/06/2008).

WAHLBERG, N., LENEVEU, J., KODANDARAMAIAH, U., PEÑA, C., NYLIN, S., FREITAS, A.V.L. \& BROWER, A.V.Z. 2009. Nymphalid butterflies diversify following near demise at the Cretaceous/Tertiary boundary. Proc. R. Soc. B 276:4295-4302. 